\title{
A importância do volume do misturador de solventes em HPLC gradiente
}

\author{
Álvaro José dos Santos Neto \\ Instituto de Química de São Carlos, Universidade de São Paulo - USP, \\ Av. Trabalhador São-carlense, 400, Cep 13560-970, São Carlos, SP, Brasil \\ e-mail:alvarojsn@iqsc.usp.br
}

Resumo

Com os desenvolvimentos recentes em HPLC e a complexidade dos atuais desafios enfrentados pela técnica, tem sido mais comum o uso de análises no modo de eluição em gradiente de fase móvel. O uso desses gradientes permite, por exemplo, análises mais rápidas e capazes de separar compostos com faixas mais amplas de hidrofobicidade do que nas metodologias com eluição isocrática. Por outro lado, a utilização de gradiente é mais exigente com a configuração da instrumentação analítica. Essas configurações podem ser particularmente críticas em casos de análise com corridas curtas em UHPLC ou com o uso de colunas microbore em LC-ESI-MS. Dependendo do tipo de detector e fase móvel utilizada, volumes muito pequenos de mistura podem provocar excessivo ruído e instabilidades na linha de base do cromatograma. Pelo contrário, um volume muito grande do misturador pode acarretar atrasos no efetivo início do gradiente e demora no recondicionamento da coluna ao fim da corrida cromatográfica. Nesse artigo será feita uma revisão de algumas particularidades do uso de gradiente, bem como dos respectivos cuidados e ajustes para se minimizar as possibilidades de falha nesse tipo de análise.

Palavras-chave

HPLC; UHPLC; colunas microbore; misturador de solvents; volume de atraso do gradiente; instrumentação.

\section{The importance of the solvent mixing volume in gradient HPLC}

\section{Abstract}

With the recent developments in HPLC and the complexity of the current challenges faced by this technique the use of mobile phase gradients has become more usual. The use of gradients allows, for example, fast analysis able to separate compounds with wide ranges of hydrophobicity than possible with isocratic elution. On the other hand, the use of gradient is more demanding regarding the instrumental setup. These setups can be particularly critical in the case of short chromatographic runs in UHPLC or with the use of microbore columns on LC-ESI-MS. Depending on the type of detector and mobile phase used, very low mixing volumes can lead to excessive noise in the form of ripple in the chromatographic baseline. In contrast, a very high mixing volume can cause gradient delay and extend the time needed for column equilibration at the end of the run. Here we present a revision about some particularities regarding the use of gradient, as well as the respective caution and adjustments to minimize the chances of failure in this kind of analysis.

Keywords

HPLC; UHPLC; microbore column; solvent mixing; gradient delay volume; instrumentation. 


\section{Aspectos gerais}

Apesar da maior simplicidade das análises com eluição isocrática em HPLC (aquelas em que não há variação da composição/força de eluição da fase móvel ao longo da separação), diversas aplicações requerem o uso de eluição em gradiente de fase móvel. Um exemplo seria a análise de amostras complexas para as quais se necessita reduzir o tempo de eluição de compostos que apresentam alta interação com a fase estacionária da coluna (sem prejudicar a separação dos analitos menos retidos presentes na mesma amostra). Análises em gradiente exigem instrumentação mais sofisticada e solventes mais puros, causam maiores problemas na linha de base do detector e são mais problemáticas na transferência do método entre diferentes equipamentos. Embora existam essas complicações, certas situações exigem as análises em gradiente, como amostras com compostos de interesse apresentando fatores de retenção (k) muito amplos em análise isocrática; amostras contendo interferentes muito retidos e que podem eluir tardiamente em análises subsequentes ou sobrecarregar a coluna; amostras muito diluídas e em solventes fracos, as quais necessitam pré-concentração na coluna cromatográfica.

Para a formação de gradientes em HPLC há duas técnicas mais comumente empregadas: o gradiente de baixa pressão (Low-Pressure Gradient - LPG) usando válvula proporcional de mistura precedendo a bomba ou o gradiente de alta pressão (High-Pressure Gradient - HPG) usando dois ou mais dispositivos de bombeamento. Em ambos ocorre a programação da variação da composição final da fase móvel em função do tempo, de maneira a garantir um gradiente de solventes na fase móvel. Obviamente, se necessário, tais sistemas podem operar no modo isocrático, permitindo uma fácil alteração na composição desejada para a fase móvel isocrática. As bombas mais comumente encontra- das no mercado para ambas as aplicações são a bomba ternária ou quaternária de mistura sob baixa pressão e a bomba binária de mistura sob alta pressão, usadas para LPG e HPG, respectivamente. De qualquer forma, na utilização dessas bombas ocorrem inomogeneidades localizadas na composição da fase móvel impulsionada para o restante do sistema cromatográfico, tornando necessária a existência de um adequado misturador para que a combinação dos solventes torne-se perfeitamente homogênea. Em revisão anterior, um maior detalhamento sobre o funcionamento de gradientes de alta e baixa pressão foi fornecido $^{[1]}$. Basicamente, a formação inicial da fase móvel em bomba de mistura sob baixa pressão possui a característica segmentada e é realizada antes da bomba cromatográfica propriamente dita, de maneira que a mistura entre os solventes deve atravessar o sistema de bombeamento antes de, efetivamente, chegar ao misturador do sistema. Em bomba binária de alta pressão, pelo contrário, ocorre a confluência entre os solventes impulsionados separadamente pelos dois dispositivos de bombeamento e as pequenas pulsações desses dispositivos fazem com que a composição da fase móvel não seja perfeitamente homogênea antes de uma adequada mistura. A Figura 1 ilustra as diferenças na forma de composição da fase móvel nos sistemas para LPG (Figura 1a) e HPG (Figura 1b), bem como a característica dessa fase móvel antes e após a passagem pelo misturador ${ }^{[2]}$.

Maiores detalhes sobre o escopo do uso das bombas mencionadas acima podem ser consultados na revisão técnica de GratzfeldHuesgen ${ }^{[3]}$. Em aplicações não muito exigentes, ambos os tipos de bombas (mistura de baixa ou alta pressão) apresentam desempenho adequado. Todavia, com ambas, se o misturador utilizado não for eficiente na homogeneização da fase móvel, prejuízos podem ocorrer no desempenho final da análise cromatográfica. 

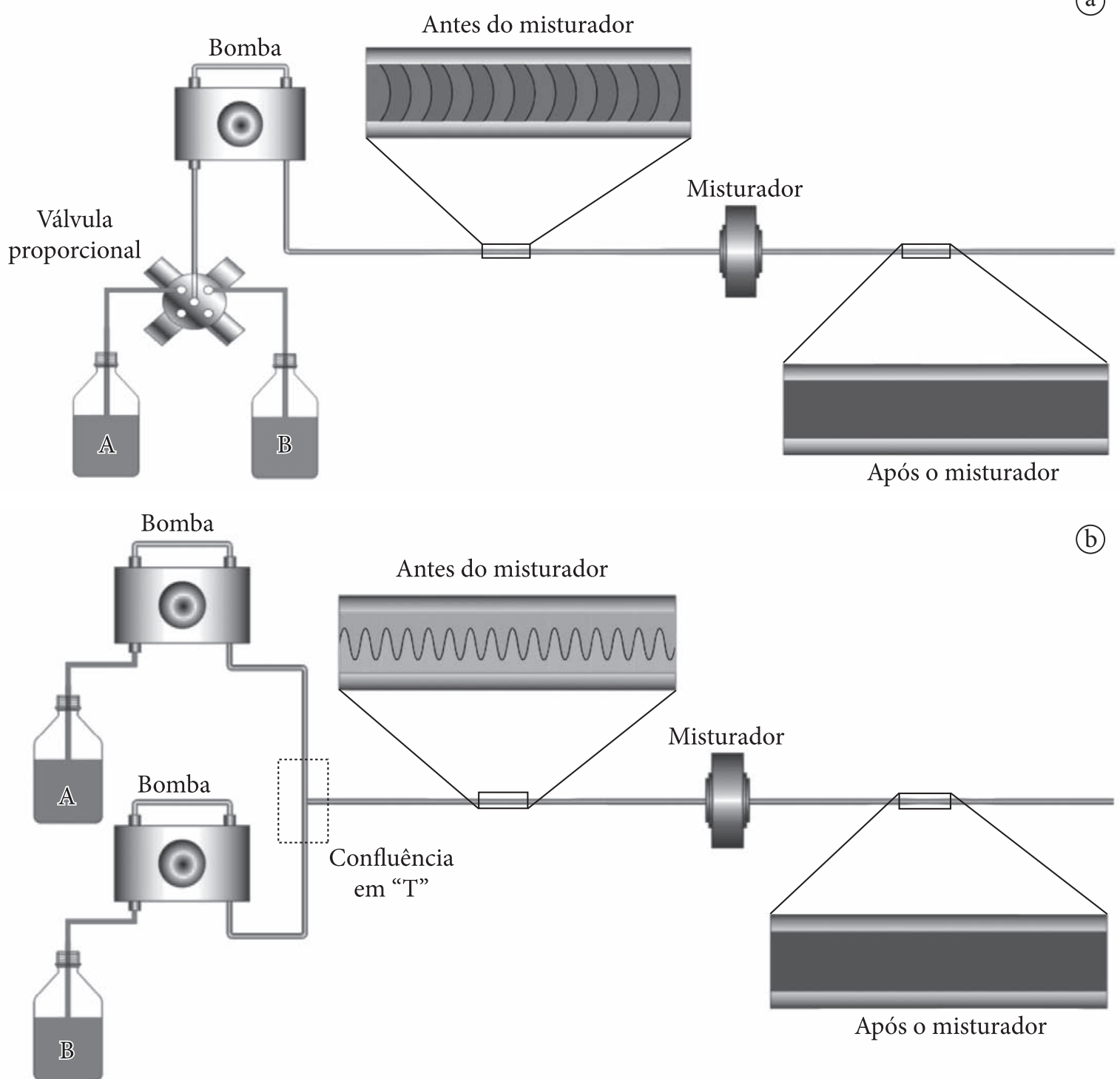

Figura 1 llustração dos sistemas de bombeamento utilizados para a) LPG; e b) HPG. Adaptação feita com permissão de Dionex (Part of Thermo Fisher Scientific) ${ }^{[2]}$.

Em casos de insuficiente volume ou ineficiência para uma adequada homogeneização, o problema mais comum é o aumento do ruído da linha de base (na forma de ondulações, ou ripple em inglês). Nesse caso, quanto mais sensível for o detector às variações na composição da fase móvel, mais críticos serão os problemas com ondulações da linha de base. Outro problema que pode ocorrer em alguns casos é a deterioração da eficiência cromatográfica. Isso pode acontecer por causa de pequenas diferenças de interação localizadas ao longo do pico cromatográfico, em função das diferentes forças de eluição encontradas na fase móvel, e que levam à deformação da banda cromatográfica.

Não menos importante do que um volume mínimo suficiente para a mistura, um volume excessivo também apresenta detrimentos às análises em gradientes, particularmente em alguns casos, como análise em UHPLC e em microbore LC-ESI-MS. Nas análises em UHPLC exploram- 
-se velocidades lineares mais altas bem como gradientes mais íngremes, de maneira a obterem-se análises cromatográficas mais rápidas. Nessas situações, um excessivo volume do misturador causaria atrasos no início efetivo do gradiente de solventes na coluna, bem como um tempo mais longo para chegar-se a um equilíbrio da coluna ao término da análise. De maneira análoga, o uso da cromatografia em escala reduzida também exige ajustes no volume utilizado para a mistura da fase móvel em questão. A Tabela 1 apresenta uma classificação para as diferentes escalas da HPLC, e, dessas, aquela que encontra maior importância é a escala microbore, a qual é extensivamente utilizada no caso do acoplamento com a espectrometria de massas utilizando a interfase de eletronebulização (electrospray) (LC-ESI-MS). Como o uso dessa abordagem vem se popularizando e, muitas vezes, instrumentos já existentes no laboratório são adaptados para essa técnica, faz-se relevante destacar a importância que ajustes sejam feitos também no volume utilizado no misturador.

O desenvolvimento de misturadores com volumes compatíveis às novas demandas e, principalmente, efetivos na homogeneização da fase móvel tem sido bastante explorado atualmente pelos fabricantes de instrumentos para HPLC. Há basicamente duas abordagens para a realização da mistura: o uso de misturadores estáticos ou dinâmicos. Os primeiros não utilizam peças móveis e são mecanicamente mais simples, apesar de explorarem sofisticadas tecnologias na sua concepção e fabricação, de maneira a garantir um máximo de mistura em um menor volume. Os misturadores dinâmicos ou ativos utilizam dispositivos móveis de agitação para a obtenção da mistura. Muitos deles são constituídos por uma câmara onde ocorre a confluência dos solventes a serem misturados e no interior da qual existe uma barra ou dispositivo similar de agitação. Apesar de garantirem uma mistura muito efetiva, esses agitadores apresentam maior necessidade de manutenção, geralmente têm maior volume total, e atualmente não são utilizados por todos os fabricantes, particularmente em casos de UHPLC e LC microbore. A Figura 2 ilustra o esquema de um agitador dinâmico desenvolvido em pesquisas do passado para a utilização em LC microbore. No caso dos agitadores estáticos muito tem sido feito ultimamente. Diversos fabricantes têm apresentado novas alternativas para a configuração desses misturadores, mostrando, algumas vezes, comparações entre os seus modelos e os dos concorrentes. Dessa forma, existem no mercado diferentes tecnologias para esses misturadores, entre as quais se podem citar o uso de: dispositivos preenchidos com microesferas de diferentes tamanhos e materiais; dispositivos que induzem a mistura por cisalhamento do solvente; diferentes dispositivos microfluídicos em múltiplas camadas; e dispositivos que combinam etapas sequenciais de mistura radial e longitudinal.

Tabela 1 Nomenclatura para classificação da cromatografia líquida realizada em diferentes escalas.

\begin{tabular}{ccc}
\hline Classificação & $\begin{array}{c}\text { Diâmetro } \\
\text { interno (i.d.) }\end{array}$ & Vazão típica \\
\hline LC convencional & $3,2-4,6 \mathrm{~mm}$ & $0,5-2,0 \mathrm{~mL} / \mathrm{min}$ \\
LC microbore & $1,5-3,2 \mathrm{~mm}$ & $100-500 \mu \mathrm{L} / \mathrm{min}$ \\
micro-LC $(\mu$-LC) & $0,5-1,5 \mathrm{~mm}$ & $10-00 \mu \mathrm{L} / \mathrm{min}$ \\
LC capilar $(\mathrm{cLC})$ & $150-500 \mu \mathrm{m}$ & $1-10 \mu \mathrm{L} / \mathrm{min}$ \\
nano-LC & $10-150 \mu \mathrm{m}$ & $10-1000 \mathrm{~nL} / \mathrm{min}$ \\
\hline
\end{tabular}

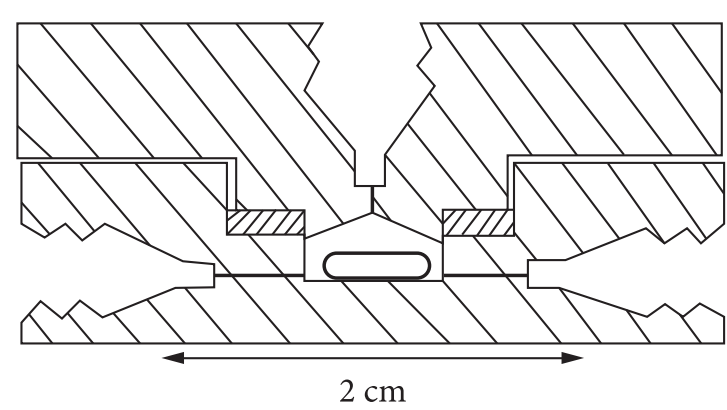

Figura 2 Esquema em corte de uma câmara de mistura dinâmica por agitação com volume interno de $80 \mu \mathrm{L}$. Com permissão, a partir da referência 4 . 


\section{Implicações do uso de volume insuficiente para mistura}

A princípio pode-se questionar o porque da necessidade de um misturador para bombas binárias de alta pressão, uma vez que a junção dos solventes em uma conexão em T aparenta ser suficiente. Logicamente bombas de alta pressão são menos críticas do que aquelas baseadas em mistura sob baixa pressão (as quais produzem um fluxo segmentado de solventes); todavia, em algumas situações, excessivo ruído é ainda observado na linha de base. Algumas bombas podem dispensar volumes tão baixos quanto $20 \mu \mathrm{L}$ ou menos por ciclo de funcionamento, dependendo se são do tipo de pistões paralelos ou sequenciais. Requer-se usualmente um volume de mistura mínimo equivalente ao volume dispensado em dois ciclos da bomba para minimizar as pequenas diferenças na composição de cada um desses ciclos de preenchimento. Para as bombas de baixa pressão, a esse volume soma-se o fato que a fase móvel chega e atravessa a bomba em um fluxo segmentado e mal pré-misturado, requerendo-se um maior volume de mistura e, por conseguinte, maiores atrasos na realização de gradientes. Esse é um grande motivo para alguns fabricantes não aconselharem a configuração de baixa pressão para executar gradientes em separações típicas de LC-ESI-MS sob baixas vazões da fase móvel. Além disso, as bombas de baixa pressão não são aconselhadas por alguns fabricantes para gradientes extremos, além da faixa de 5 a 95\% de qualquer um dos constituintes da fase móvel ${ }^{[3]}$. Igualmente, pequenas diferenças podem ocorrer na transferência de métodos entre os dois tipos de bomba, uma vez que as bombas binárias de alta pressão utilizam a compressibilidade ótima para cada um dos solventes utilizados na mistura (orgânico e aquoso), enquanto em gradiente de baixa pressão apenas uma compressibilidade pode ser utilizada para todos os canais do solvente.
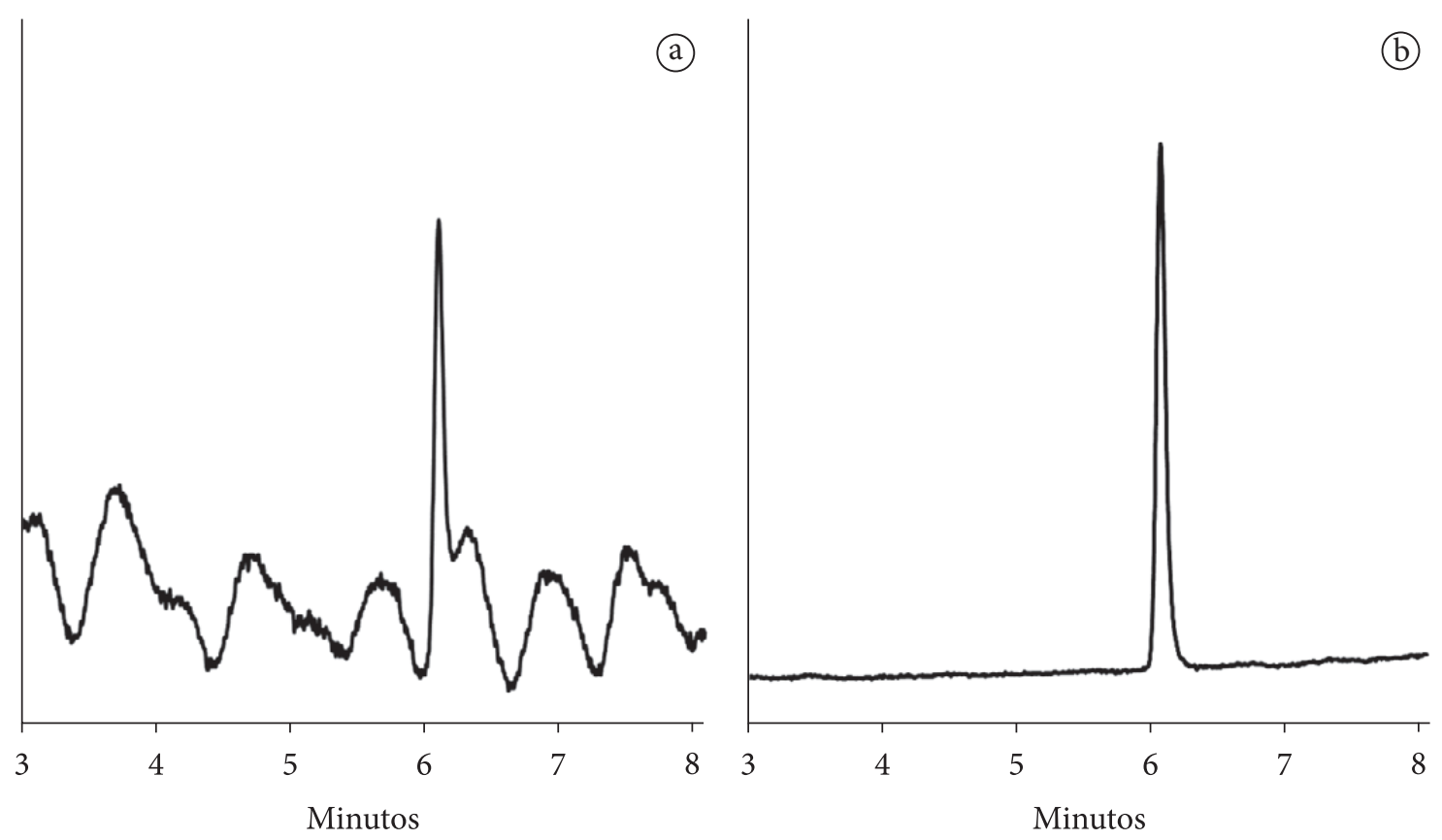

Figura 3 Cromatograma ilustrando as oscilações inadequadas causadas na linha de base por problemas na mistura do gradiente (a), em relação a um cromatograma adequado (b). Adaptação feita com permissão de Dionex (Part of Thermo Fisher Scientific) ${ }^{[2]}$. 
Os casos típicos que levam ao aparecimento de oscilações mais elevadas da linha de base são aqueles em que os solventes da mistura apresentam respostas muito diferentes no detector utilizado (por exemplo, diferente absorção no caso de detecção UV). Obviamente, o maior ruído induzido por problemas na mistura leva a maiores valores de LD e LQ nessas situações. Um trabalho de Logar e colaboradores descreve os efeitos da mistura do eluente em HPLC gradiente sobre a resposta do detector de espectrometria de lente térmica (TLS) e do UV-Vis ${ }^{[4]}$. Nesse trabalho foi feita uma comparação entre valores de $\mathrm{LD}$ obtidos em método isocrático e em gradiente para alguns compostos. Dada a maior sensibilidade do TLS às variações da composição do solvente, o uso de gradiente causou maior aumento dos valores de LD no detector TLS do que no UV-Vis, demonstrando que o primeiro sofre maior influência das inomogeneidades da mistura. A Figura 3 ilustra a diferença na razão sinal/ruído em função de uma mistura inadequada dos solventes que compõe o gradiente.

Um teste simples para a verificação de adequação da mistura pode ser feito adicionando-se metanol na linha A do gradiente e metanol contendo $0,1 \%$ de acetona na linha $\mathrm{B}$, e ajustando-se o detector UV para $260 \mathrm{~nm}$. Em seguida realiza-se uma corrida com gradiente de 0 a 100\% do componente $\mathrm{B}$ da fase móvel, verificando-se o ruído obtido na linha de base. A Figura 4 ilustra alguns experimentos em vazões típicas de LC microbore comparando diferentes tipos de misturadores.

Algumas composições de fase móvel são mais críticas em HPLC, sabe-se que a presença de ácido acético e fórmico, ou suas respectivas bases, eleva a absorção da radiação UV em baixos comprimentos de onda. Um caso tipicamente conhecido e extremo é o uso de ácido trifluoroacético (TFA) como aditivo nas fases orgânica e aquosa. Esse aditivo, em uma concentração de $0,1 \%$ em cada solvente, faz com que haja uma diferença da ordem de $160 \mathrm{mAU}$ (a $220 \mathrm{~nm}$ ) entre as absorbâncias observadas na fase $100 \%$ aquosa e $100 \%$ orgânica (acetonitrila). Neste último caso, o maior volume possível de mistura deve ser usado se o objetivo principal for a obtenção dos menores limites de detecção em detectores espectrofotométricos.

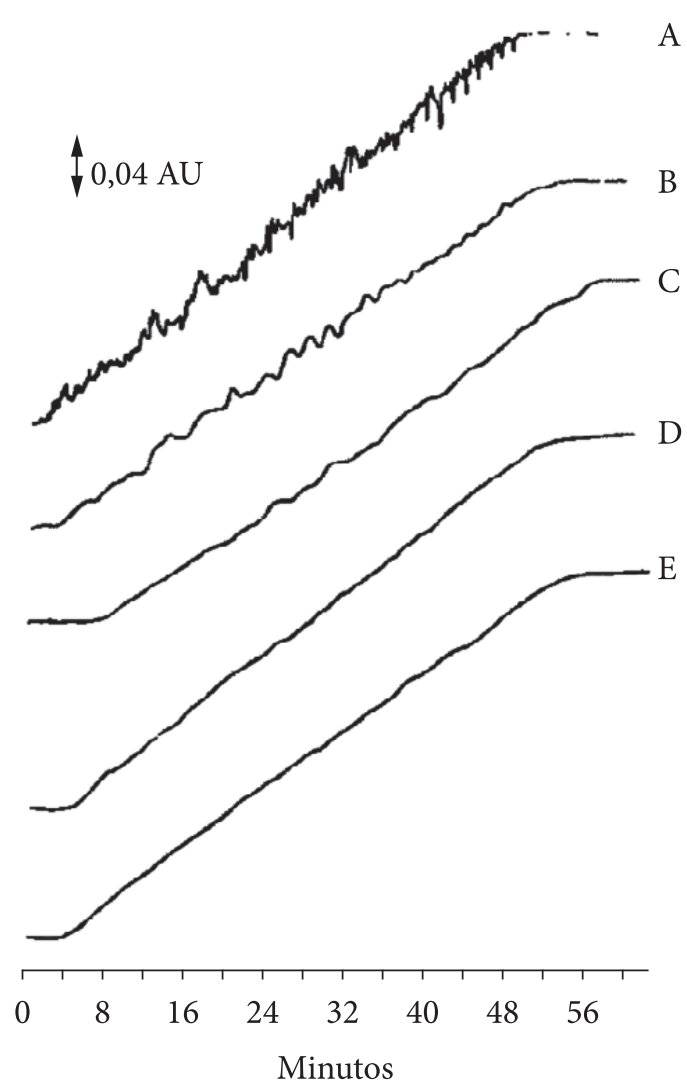

Figura 4 Testes de gradientes obtidos com metanol e metanol fortificado com acetona. a) Apenas um T misturador, b) T com tubo misturador estático de $80 \mu \mathrm{L}$, c) $\mathrm{T}$ com tubo misturador de $320 \mu \mathrm{L}$, d) misturador dinâmico de $80 \mu \mathrm{L}$, e e) T com tubo misturador estático de $80 \mu \mathrm{L}$ e misturador dinâmico de $80 \mu \mathrm{L}$. Gradiente de 0 a $100 \%$ em 50 minutos (2\%/min), vazão de fase móvel de $50 \mu \mathrm{L} / \mathrm{min}$ e misturador ligado diretamente ao detector sem o uso de coluna cromatográfica. Com permissão, a partir da referência 4 . 
Uma situação adicional é que os volumes inadequados de mistura, além de induzirem às ondulações na linha de base, também acarretam distorções no cromatograma. O exemplo da Figura 5 demonstra que mesmo em separações isocráticas usando a mistura dos solventes no sistema, uma mistura incompleta além de elevar o ruído da linha de base também distorce o perfil cromatográfico. Pode-se observar pelo cromatograma da Figura 5b que uma menor eficiência foi obtida no caso da mistura inadequada, além do já esperado aumento no ruído da linha de base.

Por fim, conforme destacado na referência 2, a própria coluna cromatográfica tem a capacidade de amplificar o ruído (ondulação) da linha de base atribuído a problemas de mistura da fase móvel. Em experimentos em que se comparam o uso de uma união, em substituição à coluna cromatográfica, pode-se observar o efeito dessa ampliação (Figura 6). Nesse caso, com o uso de um misturador de $35 \mu \mathrm{L}$, as ondulações equiva- leram a $0,26 \%$ da escala usando-se uma união e $11,1 \%$ usando-se uma coluna (uma amplificação de 43 vezes). No caso do uso de um misturador com $400 \mu \mathrm{L}$, os ruídos foram, respectivamente, 0,03 e $1,45 \%$ da escala do detector; apesar de um nível mais baixo de ruído, a amplificação é superior a 50 vezes.

Apesar de algumas aplicações serem críticas com relação a uma perfeita mistura da fase móvel, em outros casos permite-se uma considerável redução do volume de mistura. Algumas aplicações em LC-MS permitem o uso de volumes mínimos de mistura, de maneira a possibilitarem-se gradientes e re-equilíbrios mais rápidos.

\section{Implicações da existência de volume excessivo}

Se um volume inadequadamente pequeno de mistura pode ser um problema, por outro lado, um volume muito grande também não é
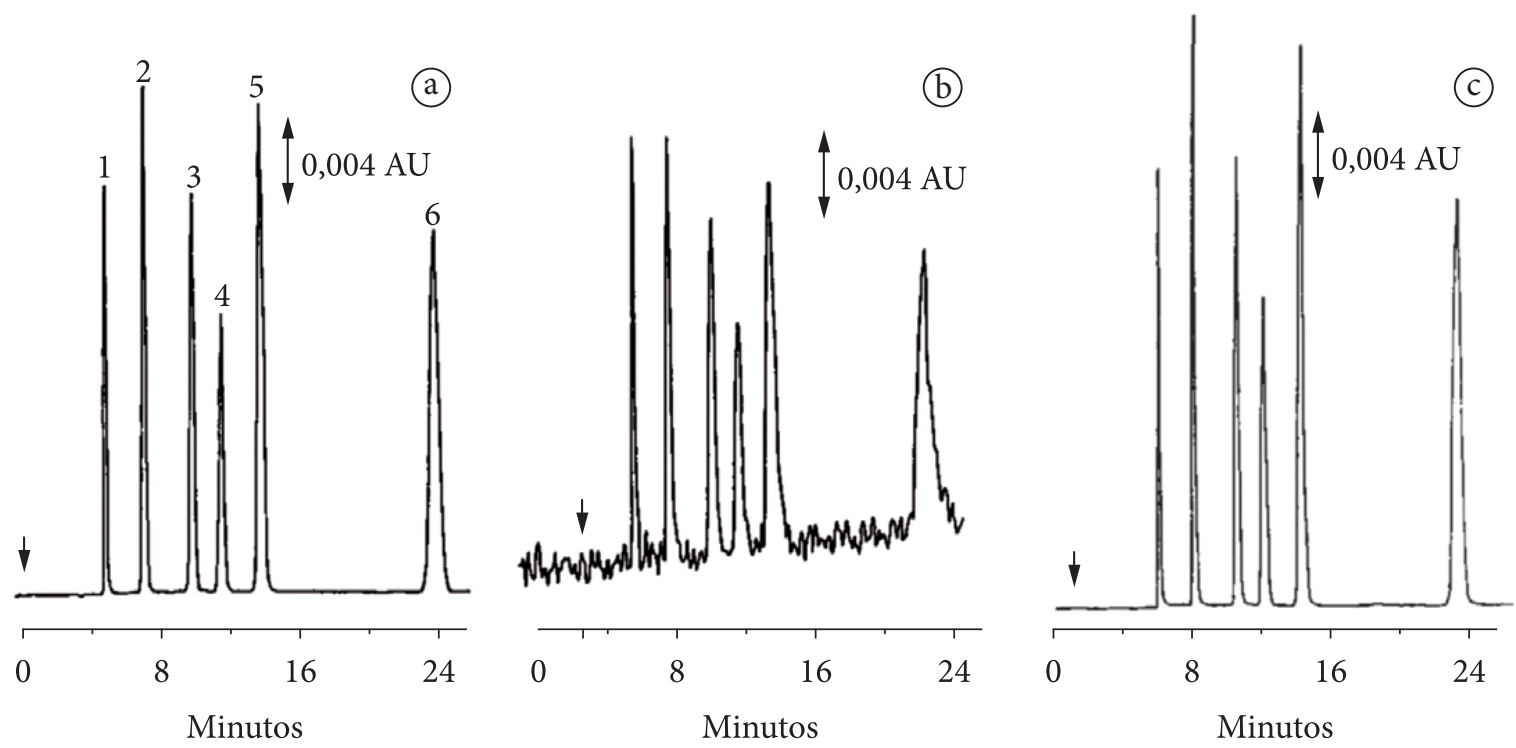

Figura 5 Separação isocrática com metanol:água (70:30, v/v). Picos 1 a 6: uracila, fenol, acetofenona, nitrobenzeno, metilbenzoato e tolveno, respectivamente. a) Fase móvel pré-misturada usando apenas uma bomba; b) Metanol e água impulsionados por duas bombas e combinados em um misturador em T. c) Metanol e água impulsionados por duas bombas e combinados em um misturador completo. Com permissão, a partir da referência 4 . 


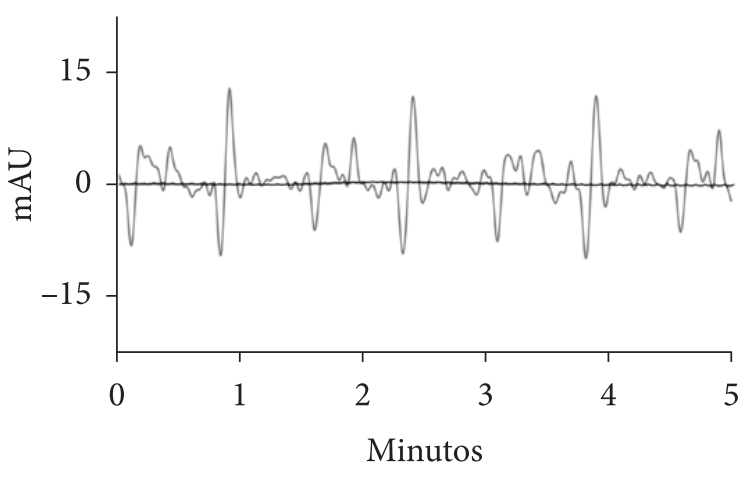

Figura 6 Comparação das oscilações da linha de base com a presença da coluna (cinza) e sem a coluna (preto). Misturador de $35 \mu \mathrm{L}$, solvente A: água/ acetonitrila 99:1 + 0,1\% TFA, solvente B: acetonitrila $100 \%+0,1 \%$ TFA, coluna: Dionex Acclaim RP C18, $3 \mu \mathrm{m}, 120 \AA, 250 \times 3,0 \mathrm{~mm}$, vazão: $1,00 \mathrm{~mL} / \mathrm{min}$, temperatura: $35^{\circ} \mathrm{C}$, comprimento de onda: $220 \mathrm{~nm}$. No experimento sem coluna foi utilizado um capilar de sílica fundida de $50 \mu \mathrm{m}$, gerando uma pressão de 400 bar. Adaptação feita com permissão de Dionex (Part of Thermo Fisher Scientific) ${ }^{[2]}$.

desejável na maioria das análises em gradiente. O problema relacionado a um volume exagerado de mistura é que esse causará atrasos na chegada do gradiente até a coluna, podendo ainda causar significativas distorções do gradiente, além de prolongar o tempo de equilíbrio entre as corridas. No caso de UHPLC, deseja-se um baixo volume de mistura, para permitirem-se gradientes rápidos e pequeno tempo de re-equilíbrio. Duas situações se destacam atualmente na questão dos ajustes necessários em sistemas HPLC não otimizados para a compatibilização com análises em gradientes. Um dos casos relevantes é a utilização de colunas para análises cromatográficas rápidas (por exemplo, colunas com tecnologia de partículas superficialmente porosas), o outro envolve o uso de colunas microbore para LC-ESI-MS, as quais tipicamente trabalham em vazões inferiores a 0,2-0,3 $\mathrm{mL} / \mathrm{min}$. Como nesses casos os gradientes utilizados são geralmente rápidos e as vazões, baixas, qualquer volume excessivo de mistura pode causar detrimentos na separação.

A Figura 7 compara análises sequenciais realizadas com um tempo de equilíbrio insuficiente entre as corridas, dadas as baixas vazões em contrapartida a um misturador com mais do que $500 \mu \mathrm{L}$ de volume. Nesse caso, a primeira corrida (com coluna equilibrada) difere das demais, tanto nos tempos de retenção quanto nas eficiências, justamente pelo fato do solvente não se re-equilibrar adequadamente no interior da coluna antes de uma nova injeção de amostra. Observa-se que na primeira corrida (cromatograma de cima) os picos são mais estreitos e eluem em tempos diferentes daqueles dos demais cromatogramas abaixo, comprovando que após a primeira corrida a coluna não consegue retornar ao seu equilíbrio inicial antes da próxima injeção. Nesse caso usou-se uma coluna com tecnologia de partículas superficialmente porosas e com diâmetro interno de 2,1 mm, para facilitar o acoplamento LC-ESI-UV-MS, e verificou-se que o volume do misturador era inadequado à condição analítica utilizada, a qual usava vazão de $0,15 \mathrm{~mL} / \mathrm{min}$.

É óbvio que não basta minimizar apenas os volumes, fato esse que leva os fabricantes de HPLC ao desenvolvimento constante de melhores tecnologias de mistura para atender os diferentes volumes demandados. Faz-se importante mencionar que não apenas o volume do misturador pode causar atrasos nos gradientes como também alguns injetores automáticos, os quais utilizam grandes alças de amostragem que permanecem no caminho do solvente entre o sistema de bombeamento e a coluna. Alguns desses instrumentos permitem a configuração dessa alça em modo by-pass depois de determinado tempo de esvaziamento, fazendo com que o gradiente não precise atravessá-la durante toda 

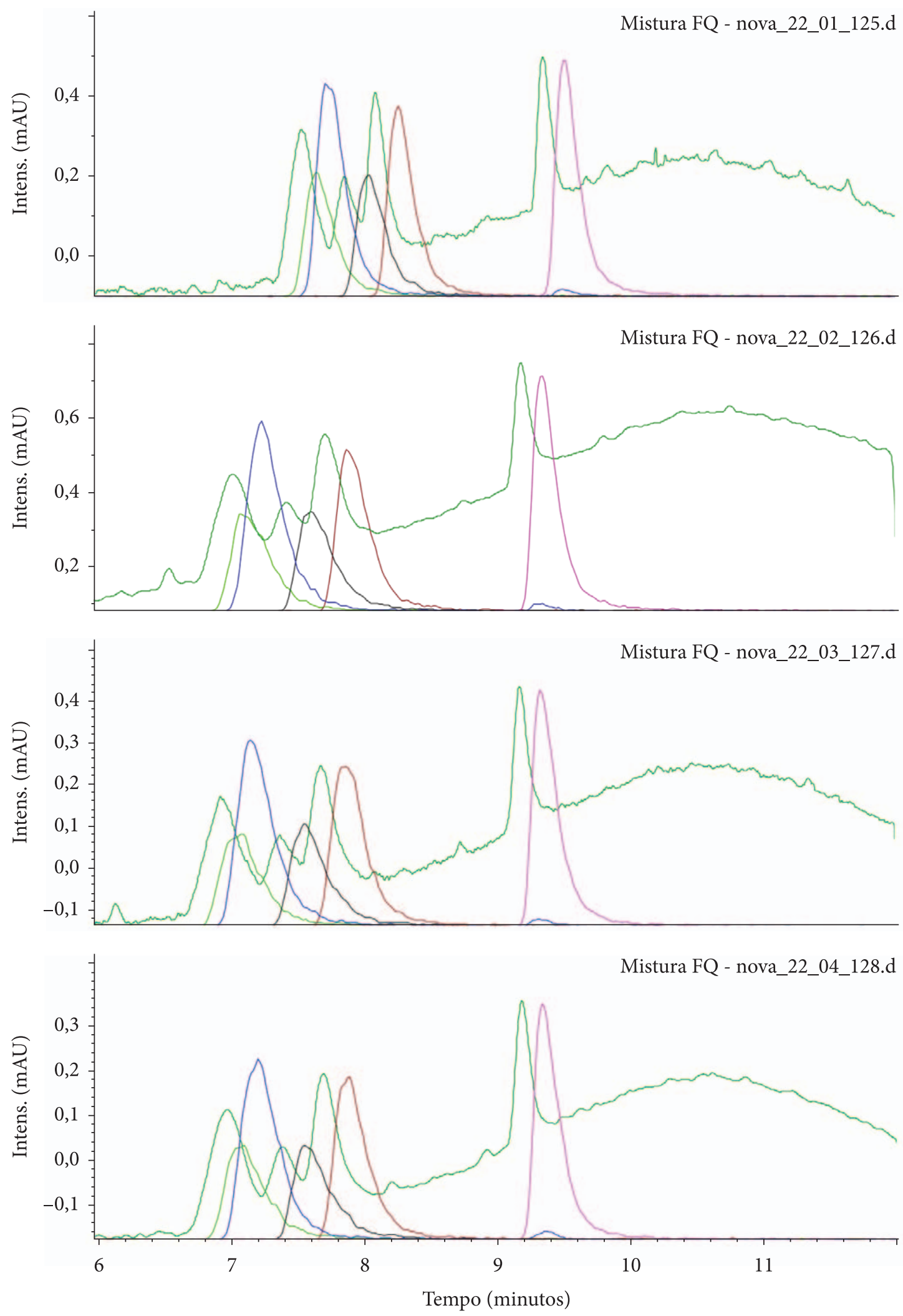

Figura 7 Comparação entre cromatogramas obtidos por LC-ESI-UV-MS com coluna microbore $(150 \times 2,1 \mathrm{~mm}$, $2,6 \mu \mathrm{m})$. Injeções sequenciais de mistura contendo 5 antibióticos. Análise em gradiente linear de 8 a $30 \%$ do solvente $B$, retornando a $8 \%$ em intervalo de 1 minuto e mantendo-se equilibrado por mais 5 minutos, em vazão de $0,15 \mathrm{~mL} / \mathrm{min}$. 
a análise. Em outros equipamentos nos quais a opção by-pass não é configurável, recomenda-se a redução do volume da alça de injeção para realização de análises suscetíveis a atrasos indesejados do gradiente.

\subsection{Como aferir e ajustar o volume adequado para o gradiente}

Diante de todo o exposto, faz-se importante saber como estimar o tempo real de atraso da chegada do gradiente à coluna, em função do volume de mistura e dos demais dispositivos utilizados. Esse atraso é útil no cálculo do denominado GDV (do inglês, gradient delay volume), o qual é o produto do tempo de atraso do gradiente com a vazão da fase móvel utilizada. Conforme

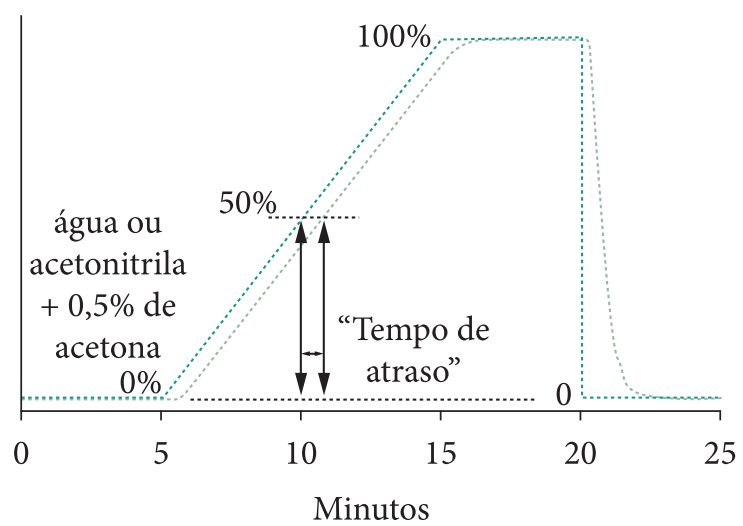

Figura 8 Determinação experimental do tempo de atraso do gradiente. Adaptação feita com permissão de Dionex (Part of Thermo Fisher Scientific) ${ }^{[2]}$.

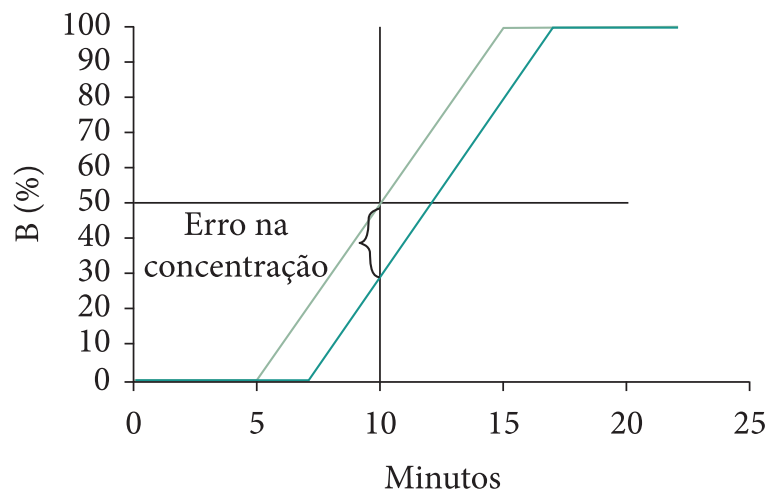

ilustrado na Figura 8, um gradiente entre $0 \mathrm{e}$ $100 \%$ do solvente B pode ser realizado em um intervalo de 10 minutos (sendo que o solvente $\mathrm{B}$ contém $0,5 \%$ de acetona em relação ao solvente A). A linha de base deve ser registrada por um detector UV em aproximadamente $260 \mathrm{~nm}$ e a distância horizontal entre o gráfico do gradiente e o da linha de base na altura de $50 \%$ de B corresponde ao tempo de atraso do gradiente.

Além do tempo de atraso no gradiente, outro parâmetro importante é a dependência que o erro na concentração (CE) da fase móvel apresenta em função da inclinação do gradiente. A Figura 9 demonstra que para um mesmo tempo de atraso do gradiente há o dobro do erro na concentração quando se duplica a inclinação do gradiente. $\mathrm{O}$ erro na concentração é:

$$
C E=\frac{\text { rampado gradiente } \times \text { volume de mistura }}{\text { vazãoda fase móvel }}
$$

A simulação de diferentes volumes de mistura demonstra claramente que um volume excessivo causa distorções no gradiente devido às dispersões no sistema. Essas dispersões causam um comportamento não linear do gradiente em função da diluição exponencial que ocorre nos extremos do gradiente. O trabalho de Powley e colaboradores descreve como realizar uma simulação do efeito do volume de mistura e demais parâmetros sobre o perfil do gradiente $^{[5]}$. Tal

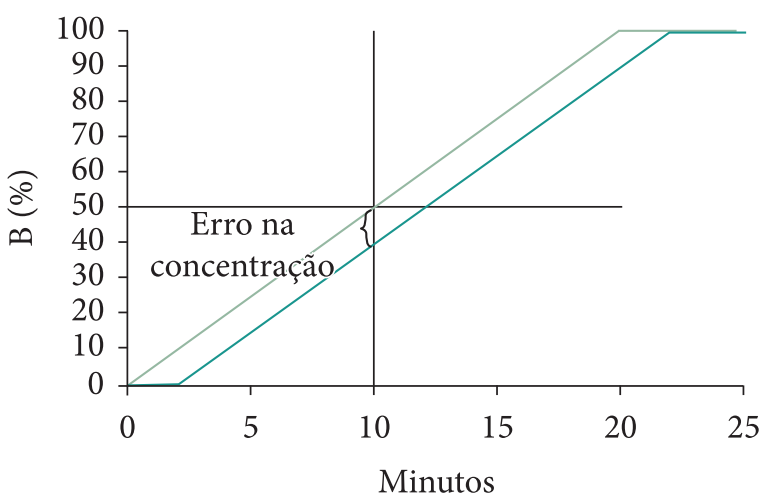

Figura 9 Determinação experimental do erro da concentração do gradiente. 
abordagem é de interesse quando se trabalha com baixas vazões de fase móvel e necessita-se saber os ajustes necessários e influências existentes sobre a transferência de métodos. A Figura 10 ilustra algumas simulações, em comparação a um sistema teórico com volume de mistura igual a zero.

Como pode ser observado na Figura 10, o aumento no volume de mistura prejudica a linearidade da variação do gradiente que realmente chega à coluna. Na simulação de um volume de $400 \mu \mathrm{L}$ de mistura, o qual causa um atraso de 2 minutos no gradiente, o erro de concentração é de $10 \%$. Esse erro mantém-se linearmente em $10 \%$ ao longo da região central do gradiente, todavia, nos extremos do gráfico há uma perda de linearidade seguindo um comportamento exponencial. No caso da mistura em $400 \mu \mathrm{L}$ há uma perda da linearidade do gradiente abaixo

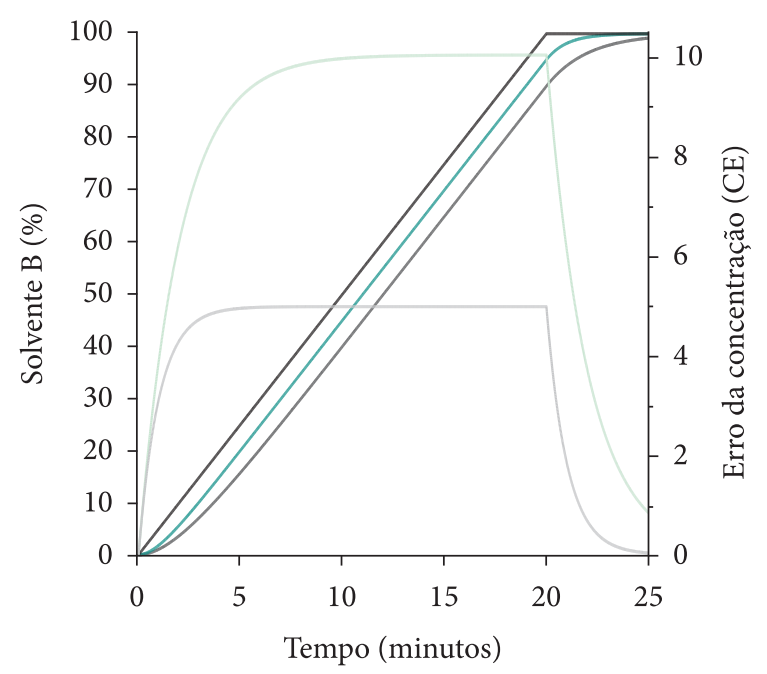

$$
\begin{aligned}
& \text { - Teórico (volume zero) - Volume de } 200 \mu \mathrm{L} \\
& \text { — Volume de } 400 \mu \mathrm{L} \\
& \text { - CE para } 400 \mu \mathrm{L}
\end{aligned}
$$

Figura 10 Simulação das distorções causadas pela dispersão no volume de mistura. Perfil teórico considerando volume zero e em comparação com 200 e $400 \mu \mathrm{L}$ de volume de mistura. Gradiente entre 0 e $100 \%$ de $B$ de 0 a 20 minutos (5\%/min) e vazão de $0,2 \mathrm{~mL} / \mathrm{min}$. de aproximadamente $10 \%$ de $\mathrm{B}$ e acima de aproximadamente $90 \%$ de B. Essas distorções são capazes de afetar a eluição dos compostos retidos nessa faixa do gradiente e devem ser levadas em consideração nos ajustes do método e principalmente na transferência da metodologia entre instrumentos com volumes diferentes.

\section{Considerações finais}

A existência de um misturador efetivo para a homogeneização da fase móvel obtida em sistemas cromatográficos com múltiplos canais de solventes é de extrema importância em diversas aplicações, quer seja usando análise isocrática ou em gradiente, com bomba binária de alta pressão ou quaternária de baixa pressão. A influência de uma adequada mistura recai sobre o ruído da linha de base, permitindo limites de detecção significativamente melhores para alguns detectores e diversas composições de fase móvel.

Um perfeito ajuste do volume utilizado para a mistura é importante para UHPLC e LC-ESI-MS, não apenas no sentido de garantir-se uma mistura minimamente aceitável para a fase móvel como também para evitar grandes atrasos no gradiente, os quais podem inviabilizar o desenvolvimento de análises rápidas, eficientes e reprodutíveis.

Em linhas gerais, para aplicações de UHPLC e LC-ESI-MS, com detectores que sofrem pouco com as irregularidades da composição do solvente (Corona CAD, ELSD e MS) podem-se utilizar misturadores pequenos, da ordem de 35 a $100 \mu \mathrm{L}$. Misturadores de tamanho intermediário (200 a $400 \mu \mathrm{L}$ ) podem ser mais adequados para minimizar as ondulações da linha de base em detector UV-Vis, sendo que o máximo de estabilidade pode ser conseguido com misturadores de até $800 \mu \mathrm{L}$. Para as aplicações mais exigentes, tais como aquelas envolvendo o uso de TFA e bom- 
bas com misturador de baixa pressão, volumes de $800 \mu \mathrm{L}$ ou mesmo superiores a $1,5 \mathrm{~mL}$ podem ser necessários.

Em vista da discussão apresentada, torna-se evidente que instrumentos convencionais não otimizados para UHPLC e LC-ESI-MS em escala microbore, muito comuns nos laboratórios de análise, necessitam ajustes para operar adequadamente em condições de gradientes. Alguns desses equipamentos podem ter misturadores com volumes ajustáveis, mas provavelmente não serão capazes de tê-los reduzidos aos níveis mais baixos desejados, ao menos sem a aquisição de novos misturadores com tecnologias e volumes mais compatíveis com as atuais exigências.

\section{Referências}

1 Santos-Neto AJ. Uma visão técnica para a compreensão e resolução de problemas em sistemas de cromatografia líquida. Scientia Chromatographica 2009; 1(1):49.
Dionex - Part of Thermo Fisher Scientific. Reliable solvent mixing in UHPLC. [cited 2011 nov.]. Dionex; 2011. Dionex Technical Note, n. 108. Available from: http:// www.dionex.com/en-us/webdocs/110921-TN108SolventMixing-UHPLC-19Aug2011-LPN2851-01.pdf.

3 Gratzfeld-Huesgen A. Scope of low and high pressure mixing. [cited 2011 nov.]. Agilent Technical Overview; 2011. Available from: http://www.chem. agilent.com/Library/technicaloverviews/Public/59907143EN.pdf.

4 Logar JK, Šikovec M, Malej A, Franko M. The effects of eluent mixing on TLS detection in gradient elution HPLC. Analytical and Bioanalytical Chemistry 2002; 374:323. PMid:12324856. http:// dx.doi.org/10.1007/s00216-002-1470-0

5 Powley CR, Howard WA, Rogers LB. Mixing considerations in the development of a gradient microbore high-performance liquid chromatographic system. Journal of Chromatography 1984; 229:43. http://dx.doi.org/10.1016/S0021-9673(01)97820-3

Recebido: 15/11/2011

Aceito: 17/11/2011 